Quaestiones Mathematicae 29(2005), 59-72.

(c) 2005 NISC Pty Ltd, www.nisc.co.za

\title{
THICK SETS IN BANACH SPACES AND THEIR PROPERTIES
}

\author{
Olav NygaARD \\ Department of Mathematics, Agder University College, Servicebox 422, \\ 4604 Kristiansand, Norway. \\ E-Mail olav.nygaard@hia.no http://home.hia.no/ºlavn/
}

\begin{abstract}
We make a survey over results involving the concepts thick and $w^{*}$-thick set and provide a lot of known such sets. We show that the long standing "separable quotient problem" and a problem from function theory are closely connected to thickness.
\end{abstract}

Mathematics Subject Classification (2000): Primary: 46B20.

Key words: Thick set, $w^{*}$-thick set.

1. Definitions and general results. Kadets and Fonf $[\mathrm{KF}]$ defined a set in a Banach space to be thin if it can be represented as a countable non-decreasing union of non-norming sets. (A set in a Banach space is said to be non-norming if its closed absolutely convex hull does not contain any ball centered at the origin.) As in [N1] and [N2], let us say that a set is thick if it is not thin. One has the following "omnibus-theorem" describing thick sets (here recall that $T: Y \rightarrow X$ Tauberian means $\left(T^{* *}\right)^{-1}(X) \subset Y$ and note that for Tauberian operators $T B_{Y}$ is closed in $X)$ :

Theorem 1.1. Let $A$ be a subset of a Banach space $X$. The following assertions are equivalent.

(a) The set $A$ is thick.

(b) Whenever a family of continuous linear operators from the space $X$ to some Banach space is pointwise bounded on $A$, then this family is norm bounded.

(c) Whenever a sequence of functionals in the dual space $X^{*}$ is pointwise bounded on $A$, then this sequence is norm bounded.

(d) Whenever $Y$ is a Banach space and $T: Y \rightarrow X$ is a continuous linear operator such that $T Y \supset A$, then $T Y=X$.

(e) Whenever $Y$ is a Banach space and $T: Y \rightarrow X$ is a continuous linear Tauberian injection with $T^{* *}$ also a Tauberian injection, such that $T Y \supset A$, then $T Y=X$

(f) The span of $A$ is dense and barrelled. 
(g) Whenever $(\Omega, \Sigma, \mu)$ is a measure space and a $w^{*}$-measurable function $g: \Omega \rightarrow$ $X^{*}$ is such that, for all $x \in A, x \circ g \in L_{1}(\mu)$, then $g$ is $w^{*}$-integrable.

Proof. Let us give the main ingredients of a proof. We first concentrate on $(\mathrm{a}) \Rightarrow$ (b) $\Rightarrow(\mathrm{c}) \Rightarrow(\mathrm{a})$. Then we look at $(\mathrm{a}) \Rightarrow(\mathrm{d}) \Rightarrow(\mathrm{e}) \Rightarrow(\mathrm{a})$. After that we explain (f) $\Leftrightarrow$ (b) and end the proof with (a) $\Leftrightarrow(\mathrm{g})$. But at the very start, observe that a set $W \subset X$ is non-norming if and only if its absolutely convex hull is non-norming if and only if $\inf _{x^{*} \in S_{X^{*}}} \sup _{x \in W}\left|x^{*}(x)\right|=0$.

First the chain (a) $\Rightarrow(\mathrm{b}) \Rightarrow(\mathrm{c}) \Rightarrow(\mathrm{a})$ : Let $Y$ be some Banach space and denote as usual by $\mathcal{L}(X, Y)$ the space of bounded linear operators from $X$ to $Y$. Assume the family $\Gamma \subset \mathcal{L}(X, Y)$ is pointwise bounded on $A$ and put

$$
A_{n}=\{x \in A:\|T x\| \leq n \text { for all } T \in \Gamma\} .
$$

By the pointwise boundedness $A=\cup_{n} A_{n}$, an increasing, countable union. Since $A$ is thick, for some $m$ there is a $\delta>0$ such that the absolute convex hull $\mathcal{A}_{m}$ of $A_{m}$ contains $\delta B_{X}$. In other words $(1 / \delta) \mathcal{A}_{m}$ contains $B_{X}$. Now

$$
\sup _{x \in B_{X}}\|T x\| \leq \frac{1}{\delta} \sup _{x \in \mathcal{A}_{m}}\|T x\| \leq \frac{m}{\delta},
$$

so $\Gamma$ is bounded by $m / \delta$. Note that this is more or less the same argument as the standard proof of the Banach-Steinhaus theorem. That (b) $\Rightarrow$ (c) is obvious so we turn to $(\mathrm{c}) \Rightarrow(\mathrm{a})$. For this assume $A$ is thin. Then we can find an increasing, countable family of non-norming sets $\left(A_{n}\right)$ such that $A=\cup_{n} A_{n}$. Since $A_{1}$ is nonnorming, we can find $y_{1}^{*}$ with $\left\|y_{1}^{*}\right\|=1$ but $\sup _{x \in A_{1}}\left|y_{1}^{*}(x)\right|<2^{-1}$. Since $A_{2}$ is norming we pick $y_{2}^{*}$ with $\left\|y_{2}^{*}\right\|=1$ but $\sup _{x \in A_{2}}\left|y_{2}^{*}(x)\right|<2^{-2}$. We construct in this manner a sequence $\left(y_{n}^{*}\right)$ in the dual unit sphere $S_{X^{*}}$ with $\sup _{x \in A_{n}}\left|y_{n}^{*}(x)\right|<2^{-n}$. It remains to explain why the unbounded family $\left(x_{n}^{*}\right)$ given by $x_{n}^{*}=2^{n} \cdot y_{n}^{*}$ is pointwise bounded on $A$. For this, let $x$ be some point of $A$. We want to show that $\sup _{n}\left|x_{n}^{*}(x)\right|<\infty$. Find some $k$ such that $x \in A_{k}$ and remember $x \in A_{n}$ whenever $n \geq k$. There are two cases, $n<k$ and $n \geq k$. In the first case $\left|x_{n}^{*}(x)\right| \leq\left\|x_{n}^{*}\right\|\|x\|<2^{k}\|x\|<\infty$. In the second case $\left|x_{n}^{*}(x)\right|<2^{n} \cdot 2^{-n}=1$. (We will improve this simple technique in the proof of $(\mathrm{g}) \Rightarrow(\mathrm{a})$.)

Now we turn to $(\mathrm{a}) \Rightarrow(\mathrm{d}) \Rightarrow(\mathrm{e}) \Rightarrow(\mathrm{a})$. First $(\mathrm{a}) \Rightarrow(\mathrm{d})$ : Let $Y$ be a Banach space and assume $A$ is thick and $T Y \supset A$. Clearly $T Y$ also contains the absolute convex hull of $A$ so we may just as well assume $A$ is absolutely convex. We have $A=\cup_{n}\left(A \cap n \cdot T B_{Y}\right)$, a countable, increasing union of absolutely convex sets. Since $A$ is thick, there are an $m$ and a $\delta>0$ with $\overline{\left(A \cap m T B_{Y}\right)} \supset \delta T B_{X}$. In particular, $\overline{T B_{Y}} \supset(\delta / m) T B_{X}$, and the result follows from a classical result due to Banach. That $(\mathrm{d}) \Rightarrow(\mathrm{e})$ is clear, we look at $(\mathrm{e}) \Rightarrow(\mathrm{a})$. We do this proof the following way: We prove how to obtain a Banach space $Y$ and an injection $T: Y \rightarrow X$ such that $T B_{Y}$ is closed, $T Y \supset A$ but $T$ is not onto $X$ whenever $A$ is thin. After this we explain how to obtain the remaining properties in (e). Assume $A$ is thin. If $A$ is not even norming, then take $Y$ as the span of $\mathcal{A} \cap B_{X}$ with $\mathcal{A} \cap B_{X}$ as unit ball (the Banach disc of $\mathcal{A} \cap B_{X}$ ) and let $T$ be the embedding of $Y$ into $X$. If $A$ is norming the idea is to find an absolutely convex, bounded, closed set $\tilde{A}$ such that 
$\tilde{A}$ is non-norming but has at least the same span as $A$. Then we use the technique from the non-norming case. We now construct $\tilde{A}$ : Write $A=\cup_{n} A_{n}$, an increasing, countable union of non-norming sets. Note that, since $\cup_{n} A_{n}=\cup_{n}\left(A_{n} \cap n B_{X}\right)$ and since $\left(A_{n} \cap n B_{X}\right)$ is just as non-norming as $A_{n}$ is, we may assume each $A_{n}$ to sit in $n B_{X}$. Put $B_{1}=A_{1}, B_{n}=A_{n} \backslash A_{n-1}$, let $B=\cup_{n}\left(1 / n^{2}\right) B_{n}$ and denote by $\mathcal{B}$ the closed, absolute convex hull of $B$. We take $\tilde{A}$ as $\mathcal{B}$. Closedness, absolute convexity and boundedness of $\tilde{A}$ is then clear, it is also clear that the span of $\tilde{A}$ contains the span of $A$ so we need only prove that it is non-norming as well. We will prove that $\tilde{A}$ contains no set of type $\varepsilon B_{X}, \varepsilon>0$. To see this, pick an $m$ such that $1 / m<\varepsilon$ and use the non-normingness of $A_{m}$ to find an $x^{*} \in S_{X^{*}}$ with $\sup _{x \in A_{m}}\left|x^{*}(x)\right|<\varepsilon / 2$. We want to show that $\sup _{x \in \tilde{A}}\left|x^{*}(x)\right|<\varepsilon$. To show this, by linearity and continuity, it is naturally enough to show that $\sup _{x \in B}\left|x^{*}(x)\right|<\varepsilon / 2$. Take an arbitrary $x \in B$. There are two possibilities, either $x$ is in some $\left(1 / n^{2}\right) B_{n}$ for $n \leq m$ or it has to be that $x$ sits in some $\left(1 / n^{2}\right) B_{n}$ for $n>m$. In the first case, remember how $x^{*}$ was chosen,

$$
\left|x^{*}(x)\right| \leq \frac{1}{n^{2}} \sup _{y \in A_{m}}\left|x^{*}(y)\right|<\frac{\varepsilon}{2 n^{2}} \leq \frac{\varepsilon}{2} .
$$

In the second case, remember $A_{n} \subset n B_{X}$ and $\left\|x^{*}\right\|=1$,

$$
\left|x^{*}(x)\right| \leq \sup _{n>m}\left\{\frac{1}{n^{2}} \sup _{y \in B_{n}}\left|x^{*}(y)\right|\right\} \leq \sup _{n>m}\left\{\frac{1}{n^{2}} \cdot n\right\}<\frac{1}{m}<\frac{\varepsilon}{2} .
$$

Now it is clear how to obtain a Banach space $Y$ and an injection $T$ with $T B_{Y}$ closed which is onto $A$ but not onto all of $X$. The additional properties in (e) are based on an application of the Davis-Figiel-Johnson-Pełczyński procedure (see e.g. [D, p. $227]$ ) on $\tilde{A}$ and the observation that this very procedure results in an isomorphic version of $X$ if and only if the set on which it is performed is norming (see [N2, Prop. 2.5] for details).

We next explain that (f) $\Rightarrow(\mathrm{b})$. For this note that, since the span of $A$ is dense, its dual is $X^{*}$. Since it is barrelled (b) follows. We use (a) and (b) to get $(f)$. Denseness follows from (a). Barrelledness follows from (b) and the already established denseness. In both directions we used the standard result that a normed space is barrelled if and only if the uniform boundedness principle works on it.

The proof why $(\mathrm{a}) \Rightarrow(\mathrm{g})$ goes like this: Let $A \subset X$ be thick, let $(\Omega, \Sigma, \mu)$ be a measure space, and let a $w^{*}$-measurable function $f: \Omega \rightarrow X^{*}$ be such that $x \circ f \in L^{1}(\mu)$ for all $x \in A$. Denote $A_{j}=\left\{x \in A: \int_{\Omega}|x f| d \mu \leq j\right\}, j \in \mathbb{N}$. Then $A=\cup_{j=1}^{\infty} A_{j} \uparrow$, and the thickness of $A$ implies the existence of some $m \in \mathbb{N}$ and $\delta>0$ such that $\mathcal{A}_{m} \supset \delta B_{X}$. Thus it clearly suffices to show that $x \circ f \in L^{1}(\mu)$ for all $x \in \mathcal{A}_{m}$. But this follows from a standard convexity and limit argument. (Note the importance that norm-closure goes via limits of sequences).

We now end the proof by using thinness to produce a function on the natural numbers $\mathbb{N}$, with values in $X^{*}, A$-scalarly integrable but still not $X$-scalarly integrable. Denoting $f(j)=x_{j}^{*}$ the point is to obtain $\sum_{j}\left|x_{j}^{*}(x)\right|<\infty$ for all $x \in A$ but the existence of $y \in X$ such that $\sum_{j}\left|x_{j}^{*}(y)\right|$ diverges. Pick an increasing family of non-norming sets $\left(A_{j}\right)$ such that the union is $A$. We use thinness analogously to 
the construction in (c) $\Rightarrow$ (a) to obtain a sequence $\left(x_{j}^{*}\right) \subset X^{*}$ with $\left\|x_{j}^{*}\right\|=2^{j}$ but $\sup _{x \in A_{j}}\left|x_{j}^{*}(x)\right|<2^{-j}$. Note that whenever $x \in A$, then there is some $m \in \mathbb{N}$ such that $x \in A_{j}$ for all $j \geq m$, and thus

$$
\sum_{j}\left|x_{j}^{*}(x)\right|=\sum_{j=1}^{m-1}\left|x_{j}^{*}(x)\right|+\sum_{j=m}^{\infty}\left|x_{j}^{*}(x)\right| \leq \sum_{j=1}^{m-1}\left|x_{j}^{*}(x)\right|+\sum_{j=m}^{\infty} \frac{1}{2^{j}}<\infty .
$$

The same holds for any subseries. What misses now is to find a $y \in X \backslash A$ together with a subseries, still denoted by same index, such that $\sum_{j}\left|x_{j}^{*}(y)\right|=\infty$. We now start that construction: Recall that $\left\|x_{j}^{*}\right\|=2^{j}$. Thus, we can pick a sequence $\left(y_{j}\right) \subset X$ with $\left\|y_{j}\right\|=2^{-j}$ and $1 \geq\left|x_{j}^{*}\left(y_{j}\right)\right|>1-1 / 4$. Note that, by completeness of $X$, since $\sum_{j=1}^{\infty} y_{j}$ is absolutely convergent, $\sum_{j=1}^{\infty} y_{j}$ and all its subseries converges in $X$. Our $y$ will either be some $x_{i_{0}}$ or be the sum of some subseries of $\sum_{j=1}^{\infty} y_{j}$ depending on which of the following two situations that occur:

1) $\lim _{j \rightarrow \infty}\left|x_{j}^{*}\left(y_{i_{0}}\right)\right| \neq 0$ for some $i_{0} \in \mathbb{N}$;

2) $\lim _{j \rightarrow \infty}\left|x_{j}^{*}\left(y_{i}\right)\right|=0$ for all $i \in \mathbb{N}$.

In the case 1), choose an increasing sequence of indices $\left(\nu_{j}\right)$ such that, for some $\delta>0$, one has $\left|x_{\nu_{j}}^{*}\left(x_{i_{0}}\right)\right|>\delta$ for all $j \in \mathbb{N}$, and put $y=x_{i_{0}}$. In case 2), put $\nu_{1}=1$ and proceed as follows. Given indices $\nu_{1}<\nu_{2}<\ldots<\nu_{j-1}(j \in \mathbb{N}, j \geq 2)$, pick an index $\nu_{j}>\nu_{j-1}$ such that

$$
\sum_{i=1}^{j-1}\left|x_{\nu_{j}}^{*}\left(x_{\nu_{i}}\right)\right|<\frac{1}{4} \quad \text { and } \quad \frac{2^{\nu_{j-1}}}{2^{\nu_{j}}} \leq \frac{1}{2^{j+1}} .
$$

Denoting $y=\sum_{i=1}^{\infty} x_{\nu_{i}}$ (remember this series converges), it remains to observe that, whenever $j \in \mathbb{N}$ and $i>j$, one has

$$
\left|x_{\nu_{j}}^{*}\left(x_{\nu_{i}}\right)\right| \leq\left\|x_{\nu_{j}}^{*}\right\|\left\|x_{\nu_{i}}\right\| \leq \frac{2^{\nu_{i}}}{2^{\nu_{j}}} \leq \frac{2^{\nu_{i-1}}}{2^{\nu_{i}}} \leq \frac{1}{2^{i+1}},
$$

and thus, for all $j \in \mathbb{N}$,

$$
\begin{aligned}
\left|x_{\nu_{j}}^{*}(y)\right| & \geq\left|x_{\nu_{j}}^{*}\left(x_{\nu_{j}}\right)\right|-\sum_{i=1}^{j-1}\left|x_{\nu_{j}}^{*}\left(x_{\nu_{i}}\right)\right|-\sum_{i=j+1}^{\infty}\left|x_{\nu_{j}}^{*}\left(x_{\nu_{i}}\right)\right| \\
& \geq\left(1-\frac{1}{4}\right)-\frac{1}{4}-\sum_{i=j+1}^{\infty} \frac{1}{2^{i+1}} \geq \frac{1}{4} .
\end{aligned}
$$

This clearly shows that we may choose $f(j)=x_{\nu_{j}}^{*}$ and the proof is complete.

Remark 1.2. The equivalences (a) $\Leftrightarrow$ (b) $\Leftrightarrow(\mathrm{c}) \Leftrightarrow(\mathrm{d}) \Leftrightarrow(\mathrm{e})$ is in [N2]. The proofs in [N2] are again mostly slight adjustments of arguments from $[\mathrm{KF}]$. In 
$[\mathrm{BK}]$ we find the equivalences (b) $\Leftrightarrow(\mathrm{c}) \Leftrightarrow(\mathrm{d}) \Leftrightarrow$ (f). That (a) $\Leftrightarrow$ (g) is first proved in $[\mathrm{ANP}]$, but the result is perhaps implicit in [Fo1]. Concerning $(\mathrm{g}) \Rightarrow$ (a) an adjustment of the proof (done in [ANP]) shows that when $A$ is thin a scalarly $A$-integrable but not scalarly integrable $f$ into $X^{*}$ can be found for all $\sigma$-finite measure spaces.

REMARK 1.3. The technique of proving $(\mathrm{g}) \Rightarrow(\mathrm{a})$ in Theorem 1.1 gives the following result, which seems to be of its own interest (see $[\mathrm{ANP}]$ ):

Lemma 1.4. Let a subset $A \subset X$ be thin, and let $\alpha_{j} \in \mathbb{R}, \alpha_{j}>0, j \in \mathbb{N}$. Then there are $x_{j}^{*} \in X^{*}, j \in \mathbb{N}, z \in X \backslash A$, an increasing sequence of indices $\left(\nu_{j}\right)_{j=1}^{\infty}$, and a real number $\delta>0$ such that

$$
\sum_{j=1}^{\infty} \alpha_{j}\left|x_{j}^{*}(x)\right|<\infty \text { for all } x \in A
$$

but $\alpha_{\nu_{j}}\left|x_{\nu_{j}}^{*}(z)\right|>\delta$ for all $j \in \mathbb{N}$.

Let us look at a notion dual to thickness by calling a set in the dual space of a Banach space $X w^{*}$-non-norming if its $w^{*}$-closed absolutely convex hull does not contain any ball centered at the origin, and defining a set $A \subset X^{*}$ to be $w^{*}$-thin [resp. $w^{*}$-thick] if it can be represented as a countable non-decreasing union of $w^{*}$-non-norming sets [resp. if it is not thin]. From [Fo2], [N2], [ANP], [AN] and [N3] one also has an "omnibus-theorem" for $w^{*}$-thickness:

Theorem 1.5. Let $X$ be a Banach space and let $A$ be a subset of the dual space $X^{*}$. The following assertions are equivalent.

(a) The set $A$ is $w^{*}$-thick in $X^{*}$.

(b) Whenever a family of dual continuous linear operators from $X^{*}$ to some dual Banach space $Y^{*}$ is pointwise bounded on $A$, then this family is norm bounded.

(c) Whenever a sequence of elements of the space $X$ is pointwise bounded on $A$, then this sequence is norm bounded.

(d) Whenever $Y$ is a Banach space and $T: X \rightarrow Y$ is a continuous linear operator such that $T^{*} Y^{*} \supset A$, then $T^{*} Y^{*}=X^{*}$.

(e) Whenever $Y$ is a Banach space and $T: Y^{*} \rightarrow X^{*}$ is a continuous linear dual Tauberian operator such that $T^{* *}$ is also a Tauberian injection and such that $T^{*} Y^{*} \supset A$, then $T^{*} Y^{*}=X^{*}$.

(f) Whenever $(\Omega, \Sigma, \mu)$ is a measure space and a measurable function $g: \Omega \rightarrow X$ is such that, for all $x^{*} \in A, x^{*} \circ g \in L_{1}(\mu)$, then $g$ is weakly integrable.

(g) Whenever a series $\sum_{j=1}^{\infty} x_{j}$ is such that $\sum_{j=1}^{\infty}\left|x^{*} x_{j}\right|<\infty$ for every $x^{*} \in A$, then the series is weakly unconditionally convergent, that is $\sum_{j=1}^{\infty}\left|x^{*} x_{j}\right|<\infty$ for every $x^{*} \in X^{*}$. 
(h) Whenever $\mathcal{F}$ is an algebra of subsets of a set $\Omega$ and $F: \Omega \rightarrow X$ is a vector measure such that $x^{*} F$ is of bounded variation for every $x^{*} \in A$, then $F$ is bounded.

(i) Whenever $\Omega \subset \mathbb{C}$ is open and $f: \Omega \rightarrow X$ is such that $x^{*} \circ f$ is holomorphic for every $x^{*} \in A$, then $f$ is holomorphic.

Proof. Much is similar to the proof of Theorem 1.1, however some modifications have to be made. In $(\mathrm{a}) \Rightarrow(\mathrm{b})$, denote the family of operators by $\Gamma$ and put $A_{n}=\left\{x^{*} \in A:\left\|T^{*} x^{*}\right\| \leq n\right.$ for all $\left.T \in \Gamma\right\}$. For some $m$ the $w^{*}$-closed absolute convex hull $\mathcal{A}_{m}^{*}$ of $A_{m}$ contains some $\delta B_{X^{*}}$. We obtain for $T \in \Gamma$

$$
\sup _{x^{*} \in B_{X^{*}}}\left\|T^{*} x^{*}\right\| \leq \frac{1}{\delta} \sup _{x^{*} \in \mathcal{A}_{m}^{*}}\left\|T^{*} x^{*}\right\|,
$$

and $w^{*}$-continuity of $T^{*}$ now gives

$$
\sup _{x^{*} \in B_{X^{*}}}\left\|T^{*} x^{*}\right\| \leq \frac{m}{\delta} .
$$

(b) $\Rightarrow(\mathrm{c})$ is as obvious as can be and (c) $\Rightarrow(\mathrm{a})$ is completely analogous to the corresponding part in the proof of Theorem 1.1.

For $(\mathrm{a}) \Rightarrow(\mathrm{d})$ without loss of generality assume $A$ is absolutely convex, put $A_{n}=A \cap T^{*}\left(n B_{Y^{*}}\right)$ and observe that by thickness, for some $m$ the $w^{*}$-closure of $A_{m}$ contains some $\delta B_{X^{*}}$. In particular, the $w^{*}$-closure of $T^{*}\left(m B_{Y^{*}}\right)$ contains $\delta B_{X^{*}}$. By $w^{*}$-continuity of $T^{*}$ and Alaoglu's theorem used on $B_{Y^{*}}, T^{*}\left(m B_{Y^{*}}\right)$ itself contains $\delta B_{X^{*}}$ and so $T^{*}\left(B_{Y^{*}}\right) \supset(\delta / m) B_{X^{*}}$. Concerning (e) $\Rightarrow$ (a) the technique that was done in the proof of Theorem 1.1 of transferring a norming thin set into a non-norming set with at least the same span works completely analogously for $w^{*}$-norming $w^{*}$-thin sets, but the situation when the $w^{*}$-thin set $A$ is not even $w^{*}$-norming needs a comment. We may again form the Banach disc of the $w^{*}$-closed absolute convex hull $\mathcal{A}^{*}$ of $A$, but we don't know whether this becomes a dual space and the embedding is a dual operator. However, using the relatively simple observation that the Davis-Figiel-Johnson-Pełczyński procedure performed on a $w^{*}$-closed absolutely convex set results in a dual space with the embedding a dual operator (see [N3, Proposition 2.1] for a proof of this) solves the problem, and we arrive at a proof of $(\mathrm{e}) \Rightarrow(\mathrm{a})$.

(a) $\Rightarrow$ (f) comes from an argument similar to the corresponding part of the proof of Theorem 1.1, but there is an important difference. The $w^{*}$-closure is not necessarily sequential, but now the measurability of $g$ takes over; by Pettis' measurability theorem (see e.g. [DU, Theorem 2 p. 42]) $g$ is essentially separable-valued. After these remarks, let us now give all the details: Let $A \subset X^{*}$ be weak*-thick, let $(\Omega, \Sigma, \mu)$ be a measure space, and let a measurable function $f: \Omega \rightarrow X$ be such that $x^{*} f \in L^{1}(\mu)$ for all $x^{*} \in A$. Denote $A_{j}=\left\{x^{*} \in A: \int_{\Omega}\left|x^{*} f\right| d \mu \leq j\right\}, \quad j \in \mathbb{N}$. Then $A=\bigcup_{j=1}^{\infty} A_{j} \uparrow$, and the $w^{*}$-thickness of $A$ implies the existence of some $m \in \mathbb{N}$ and $\delta>0$ such that $A_{m}^{*} \supset \delta B_{X^{*}}$. So it clearly suffices to show that $x^{*} f \in L^{1}(\mu)$ for all $x^{*} \in A_{m}^{*}$. Fix an arbitrary $x^{*} \in A_{m}^{*}$. Since $f$ is essentially separable valued, 
there is a sequence $\left(y_{n}^{*}\right) \subset \operatorname{absconv}\left(A_{m}\right)$ such that $y_{n}^{*} f \rightarrow x^{*} f \mu$-almost everywhere on $\Omega$; hence $x^{*} f$ is measurable. Since, for any $y^{*} \in \operatorname{absconv}\left(A_{m}\right)$, one has $\int_{\Omega}\left|y^{*} f\right| d \mu \leq m$, by Fatou's lemma also $\int_{\Omega}\left|x^{*} f\right| d \mu \leq m$ and thus $x^{*} f \in L^{1}(\mu)$. (f) of course implies (g) and the argument that $(\mathrm{g}) \Rightarrow$ (a) is completely analogous to the corresponding part of the proof of Theorem 1.1.

The proof that $(\mathrm{a}) \Rightarrow(\mathrm{h})$ is easy: Put $A_{j}=\left\{x^{*} \in A:\left|x^{*} F\right|(\Omega) \leq j\right\}, j \in \mathbb{N}$. Then $A=\bigcup_{j=1}^{\infty} A_{j} \uparrow$, and the weak*-thickness of $A$ implies that there are some $m \in \mathbb{N}$ and $\delta>0$ such that $A_{m}^{*} \supset \delta B_{X^{*}}$. Thus it clearly suffices to show that, for all $x^{*} \in A_{m}^{*}$, one has $\left|x^{*} F\right|(\Omega) \leq m$. Observing that the last inequality holds for all $x^{*} \in \operatorname{absconv}\left(A_{m}\right)$, it can be easily seen to hold also for all $x^{*} \in A_{m}^{*}$. For the converse, that is the implication $(\mathrm{h}) \Rightarrow(\mathrm{a})$, let a subset $A \subset X^{*}$ be weak*-thin. We will construct an unbounded $X$-valued vector measure $F$ on the algebra $\mathcal{F}_{\mathbb{N}}$ of finite and co-finite subsets of $\mathbb{N}$ such that $\left|x^{*} F\right|(\mathbb{N})<\infty$ for every $x^{*} \in A$. We can obtain a $w^{*}$-version of Lemma 1.4 by the same argument as in the proof of $(\mathrm{g}) \Rightarrow$ (a) in Theorem 1.1:

Lemma 1.6. Let a subset $A \subset X^{*}$ be weak ${ }^{*}$-thin, and let $\alpha_{j} \in \mathbb{R}, \alpha_{j}>0, j \in \mathbb{N}$. Then there are $x_{j} \in X, j \in \mathbb{N}, z^{*} \in X^{*} \backslash A$, an increasing sequence of indices $\left(\nu_{j}\right)_{j=1}^{\infty}$, and a real number $\delta>0$ such that

$$
\sum_{j=1}^{\infty} \alpha_{j}\left|x^{*}\left(x_{j}\right)\right|<\infty \text { for all } x^{*} \in A,
$$

but $\alpha_{\nu_{j}}\left|z^{*}\left(x_{\nu_{j}}\right)\right|>\delta$ for all $j \in \mathbb{N}$.

Applying Lemma 1.6 for $\alpha_{j}=1, j \in \mathbb{N}$, produces some $z_{j} \in X, j \in \mathbb{N}, z^{*} \in X^{*}$, and $\delta>0$ such that $\sum_{j=1}^{\infty}\left|x^{*}\left(z_{j}\right)\right|<\infty$ for all $x^{*} \in A$, but $\operatorname{Re} z^{*}\left(z_{j}\right)>\delta$ for all $j \in \mathbb{N}$ (just take $z_{j}=\frac{\frac{\partial}{z^{*}\left(x_{\nu_{j}}\right)}}{\left|z^{*}\left(x_{\nu_{j}}\right)\right|} x_{\nu_{j}}$ in Lemma 1.6). It remains to define the vector measure $F: \mathcal{F}_{\mathbb{N}} \rightarrow X$ by

$$
F(E)=\left\{\begin{aligned}
0, & \text { if } E=\emptyset \text { or } E=\mathbb{N} \\
\sum_{j \in E} z_{j}, & \text { if } 0<|E|<\infty \\
-\sum_{j \in E^{c}} z_{j}, & \text { if } 0<\left|E^{c}\right|<\infty
\end{aligned}\right.
$$

The implication $(c) \Rightarrow(i)$ is just a sharper version of Dunford's classical theorem (weakly holomorphic functions are strongly holomorphic) and the proof is verbatim except one uses (c) instead of the Banach-Steinhaus theorem at the end (a proof of Dunford's theorem can be found in [Y, p. 128]). For $(\mathrm{g}) \Rightarrow(\mathrm{c})$ we follow [AN]: For each $k$, look at the segment

$$
L_{k}=\left\{r e^{i \pi / 2 k}: \frac{1}{2 k} \leq r \leq 1\right\}, \quad k=1,2, \ldots
$$

Put open disjoint neighborhoods $V_{k}$ around $L_{k}$ such that $V_{j} \cap V_{k}=\emptyset, j \neq k$ and such that $\mathbb{C} \backslash\left(L_{k} \cup\left(\mathbb{D} \backslash V_{k}\right)\right)$ is connected, where $\mathbb{D}$ is the open unit disc. (The picture 
is that $V_{k}$ is some tiny ellipsis containing $L_{k}$ with some space also at the endpoints of the segment.) Now $L_{k} \cup\left(\overline{\mathbb{D}} \backslash V_{k}\right)$ is a compact subset of the plane and by an application of Runge's theorem we can now find a polynomial $f_{k}$ such that $\left|f_{k}\right| \geq k$ on $L_{k}$ and $\left|f_{k}\right| \leq 2^{-k}$ on $\overline{\mathbb{D}} \backslash V_{k}$. (Put an open set $U_{1}$ around $L_{k}$ inside $V_{k}$ and an open set $U_{2}$ around $\overline{\mathbb{D}} \backslash V_{k}$ not intersecting $U_{1}$. Define a holomorphic function $g_{k}$ on the open set $U_{1} \cup U_{2}$ by letting $g_{k}$ be some constant higher than $k$ on $U_{1}$ and some constant lower than $2^{-k}$ on $U_{2}$. Now apply Runge's theorem ([R1, 13.7 Theorem $]$ ) to obtain the polynomial $f_{k}$.) If (c) fails we can pick a sequence $\left(x_{n}\right) \subset X$ which is pointwise bounded on $A$, but $\lim _{n}\left\|x_{n}\right\|=\infty$. Let $b_{n}=\sup _{|z| \leq 1}\left|f_{n}(z)\right|$. We may (by possibly passing to a subsequence) assume $\sum_{n} b_{n} /\left\|x_{n}\right\|<\infty$. Define $f: \mathbb{D} \rightarrow X$ by

$$
f(z)=\sum_{n} f_{n}(z) \cdot \frac{x_{n}}{\left\|x_{n}\right\|} .
$$

We will show that $f$ contradicts (i). To see that $x^{*} \circ f$ is holomorphic for every $x^{*} \in A$ we observe that on $\overline{\mathbb{D}}$

$$
\left|f_{n}(z) \cdot \frac{x^{*}\left(x_{n}\right)}{\left\|x_{n}\right\|}\right| \leq \frac{b_{n}}{\left\|x_{n}\right\|} \sup _{k \in \mathbb{N}}\left|x^{*}\left(x_{n}\right)\right|
$$

Hence the series $\sum_{n} f(z) \frac{x^{*}\left(x_{n}\right)}{\left\|x_{n}\right\|}$ converges uniformly on $\overline{\mathbb{D}}$ and so the limit $x^{*} \circ f$ is holomorphic. However, if we choose $z_{n} \in L_{n}$ such that $z_{n} \rightarrow 0$, then

$$
\left\|f\left(z_{n}\right)\right\| \geq\left|f_{n}\left(z_{n}\right)\right|-\left\|\sum_{k \neq n} f_{k}\left(z_{n}\right) \frac{x_{k}}{\left\|x_{k}\right\|}\right\| \geq n-\sum_{k} 2^{-k}=n-1 .
$$

Hence $f$ is discontinuous at 0 .

REMARK 1.7. The equivalences (a) $\Leftrightarrow(\mathrm{b}) \Leftrightarrow(\mathrm{c}) \Leftrightarrow(\mathrm{d})$ is in [N2], again mostly slight adjustments of arguments from [KF] (see also [Fo2, Proposition 1] and the sentence introducing it). That $(\mathrm{a}) \Leftrightarrow(\mathrm{e})$ is the main result of [N3]. In [ANP] we find the equivalences (a) $\Leftrightarrow(\mathrm{f}) \Leftrightarrow(\mathrm{g}) \Leftrightarrow(\mathrm{h})$. Again, note that the equivalences (a) $\Leftrightarrow(\mathrm{f}) \Leftrightarrow(\mathrm{g})$ are more or less known from [Fo1]. That (c) $\Leftrightarrow$ (i) is in [AN, p. 781-782] but for separable spaces the result can be found as [Fo1, Theorem 4].

On the contrary to Theorem 1.1, Theorem 1.5 has nothing to do with results from the theory of barrelled spaces: it does not say anything about the equicontinuity of $w^{*}$-continuous linear functionals.

Note that, in the theory of analytic functions (see [FHS]), a set $A$ satisfying condition (c) of Theorem 1.5 is called a uniform boundedness deciding set (UBDset). In $[\mathrm{AN}]$ the expression determines boundedness is used.

Of course no countable set can be thick or $w^{*}$-thick, just by definition. Also, every set of second Baire category is thick. In some sense the interest in thickness is that one is allowed to test on sets which are smaller than second category for a lot of purposes. 
For our intuition, let us take a moment to mention two examples. First, note that the sequences on the unit ball in $\ell_{\infty}$, where the entries are 0 or 1 , form a thick but nowhere dense set. This, and much more general results are concluded from the Nikodým boundedness theorem (see e.g. [DU, p. 14]). To find a $w^{*}$-thick set which is not thick, take $X$ in $X^{* *}$ for any non-reflexive space $X$.

In Section 2 we give a survey over results on $w^{*}$-thick subsets of duals of Banach spaces. In Section 3 and 4 we use the general results from Section 1 to give equivalent reformulations of two longstanding problems, namely the "separable quotient problem" and a problem of strengthening Marshall's theorem on Blaschke products in the space of bounded holomorphic functions on the unit disc.

2. Thickness results for concrete sets. We now make a survey of known results, but let us start with a remark that might be helpful.

Proposition 2.1. A Banach space $X$ is reflexive if and only if every $w^{*}$-thick subset of $X^{*}$ is thick.

Proof. If $X$ is reflexive, then clearly every $w^{*}$-thick subset of $X^{*}$ is thick. Assume $X$ is not reflexive, take any $x^{* *} \in X^{* *} \backslash X$ and put $E=\operatorname{ker} x^{* *}$. Then it is elementary and well-known that $E$ is norm-closed and that $E \bigcap B_{X^{*}}$ is $w^{*}$-norming, in particular $E$ is $w^{*}$-dense. We now show that $E$ is $w^{*}$-thick. To see this we use the test given in Theorem 1.5 (e). Thus, let $Y$ be any Banach space and $T: X \rightarrow Y$ such that $T^{*} Y^{*} \supset E$. Since $E$ has co-dimension 1 in $X^{*}$ either $T^{*} Y^{*}=E$ or $T^{*} Y^{*}=X^{*}$. But the first case is impossible since a norm-closed subspace of $X^{*}$ which is the image of an adjoint is automatically $w^{*}$-closed and since $E$ is $w^{*}$-dense in $X^{*}$.

Corollary 2.2. If $x^{* *} \in X^{* *} \backslash X$ then $\operatorname{ker} x^{* *} \bigcap S_{X^{*}}$ is a $w^{*}$-thick subset of $X^{*}$.

2.1. $\boldsymbol{w}^{*}$-thickness of boundaries and extreme sets. Recall that a set $J \subset$ $B_{X^{*}}$ is called a James boundary if every $x \in X$ attains its norm on $J$. As an example, the set of extreme points of the dual unit ball is always a James boundary for $X$. We now present a beautiful result of V. P. Fonf:

Theorem 2.3. If a Banach space $X$ admits a $w^{*}$-thin James boundary $J$, then $X$ contains a copy of $c_{0}$. So if $X$ does not contain a copy of $c_{0}$, then any James boundary is $w^{*}$-thick.

Proof. We explain how the theorem can be proved with help of different papers.

(i) Note that the restriction of a James boundary to a subspace $Y$ is a James boundary for $Y$.

(ii) Put $J=\cup_{n} A_{n}$. By Simons' generalization of the Rainwater lemma [S], there is a sequence $\left(x_{n}\right)$ on $S_{X}$ which converges weakly to 0 . By the BessagaPełzcynski selection principle (see e.g. [D, p. 42]) $\left(x_{n}\right)$ can be assumed to be a basic sequence. Let $Y=\left[x_{n}\right]$. We look for $c_{0}$ inside $Y$. 
(iii) Let $T$ be the natural embedding of $Y$ into $X$. Put $B_{n}=T^{*}\left(A_{n}\right)$. Then show that $J^{\prime}=\cup B_{n}$ is a James boundary for $Y$.

(iv) Show that each $B_{n}$ is relatively norm-compact as done on page 489 in [Fo1]. Thus $Y$ has a $\sigma$-compact James boundary $J^{\prime}$.

(v) Use Lemma 27 in [FZ] to renorm $Y$ equivalently to have a countable James boundary $J^{\prime \prime}$.

(vi) Follow the proof of [FZ, Theorem 23] to construct a copy of $c_{0}$ inside a once more equivalently renormed version of $Y$. This copy is also a copy in $X$.

It is not essential that the set be a James boundary, as was shown by Fonf in [Fo2].

Theorem 2.4. If a Banach space $X$ contains no copy of $c_{0}$, then the $w^{*}$-exposed points of $B_{X^{*}}$ is $w^{*}$-thick.

REMARK 2.5. The above theorem may be true even if there is a copy of $c_{0}$ inside $X$. If $X=\mathcal{K}\left(\ell_{2}\right)$, the compact operators on $\ell_{2}$, then $X$ is separable, contains a copy of $c_{0}$ and the $w^{*}$-exposed points of $B_{X^{*}}$ is $w^{*}$-thick. The proof of this goes the following way: First, it is an easy application of Theorem 1.5 (c) to se that if $A$ and $B$ are $w^{*}$-thick subsets of $X^{* *}$ and $Y^{*}$, respectively, then $A \otimes B$ is a $w^{*}$-thick subset of $\mathcal{L}(X, Y)^{*}$, the dual of the bounded operators from $X$ into $Y$. From a theorem of Ruess and Stegall, see [Ru], for any pair of Banach spaces $X$ and $Y$,

$$
w^{*}-\exp B_{K(X, Y)^{*}}=w^{*}-\exp B_{X^{* *}} \otimes w^{*} \text {-exp } B_{Y^{*}} .
$$

Now the result follows from Theorem 2.3. However, any separable Banach space with a copy of $c_{0}$ inside may be renormed so that $w^{*}-\exp B_{X^{*}}$ is $w^{*}$-thin (see [Fo1]).

2.2. Massiveness of norm-attaining functionals. The set of norm-attaining functionals on the unit ball $B_{X}$ of a Banach space $X$ is, by the Bishop-Phelps theorem, always a norm-dense subset of $X^{*}$ and is, by the famous theorem of R.C. James, all of $X^{*}$ exactly when $X$ is reflexive. Let us denote the set $\Sigma\left(B_{X}\right)$. It is a dense $\mathcal{G}_{\delta}$ if $X$ has the Radon-Nikodým property and if $X$ is separable and $\Sigma\left(B_{X}\right)$ is of second category for each equivalent norm on $X$, then $X$ has the Radon-Nikodým property. From [KMS] we see that for $X=C[0,1], \Sigma\left(B_{X}\right)$ is of first category in $X^{*}$.

We want to remark that it may be even $w^{*}$-thin. Namely, let $X=c_{0}$, then $S=\Sigma\left(B_{c_{0}}\right)$ consists of the vectors in $\ell_{1}$ where only finitely many entries are nonzero. To see that $S$ is $w^{*}$-thin, look at the sequence in $c_{0}$ defined this way: $a_{1}=$ $(1,0,0,0), a_{2}=(1,2,0,0), a_{3}=(1,2,3,0)$ and so on. This unbounded sequence is pointwise bounded on $S$, and by Theorem 1.5 (c) $S$ is $w^{*}$-thin.

The above remark and results should be compared with the following result of Fonf ([Fo1]): 
Theorem 2.6. If the real Banach space $X$ does not contain a copy of $c_{0}$, then $\Sigma\left(B_{X}\right)$ is a $w^{*}$-thick set. If $X$ is separable and contains a copy of $c_{0}$, it can be equivalently renormed so that $\Sigma\left(B_{X}\right)$ is $w^{*}$-thin.

The complement of the functionals that attain their norm has a clear tendency of being big, however. The following result of Fonf and Lindenstrauss ([FL, Thm. 4.3]) is impressing:

Theorem 2.7. Let $X$ be a separable, non-reflexive space. Then the set of functionals in $X^{*}$ which do not attain their maximum on $B_{X}$ is a thick set.

3. Thickness and the separable quotient problem. The following problem is longstanding: Let $X$ be a Fréchet space. Does $X$ have a subspace $Y$ such that the quotient $X / Y$ is infinite-dimensional and separable? A survey of the story of "the separable quotient problem" for Banach spaces can be found in [Mu]. It turns out that the answer is yes for Fréchet spaces which are not Banach spaces.

It was shown by Saxon and Wilansky [SW] that the separable quotient problem has the following equivalent formulation: A Banach space $X$ has a separable infinite-dimensional quotient if and only if it contains a dense non-barrelled subspace.

We now combine with Theorem 1.1 to obtain the following equivalent formulations of the separable quotient problem.

Problem 3.1. The following questions are equivalent and open:

(a) Every Banach space has a separable infinite-dimensional quotient.

(b) Every Banach space contains a dense non-barrelled subspace.

(c) For every Banach space $X$ there exist a Banach space $Y$ and a Tauberian injection $T$ from $Y$ into $X$ such that $T$ has dense image but is not onto.

(d) For every Banach space $X$ there exists a set $A$ in $X$ and an unbounded sequence $\left(x_{n}^{*}\right)$ in $X^{*}$ such that the span of $A$ is dense in $X$ and $\left(x_{n}^{*}\right)$ is pointwise bounded on $A$.

(e) For every Banach space $X$ there exist a set $A$ in $X$, a measure space $(\Omega, \Sigma, \mu)$ and a $w^{*}$-measurable function $g: \Omega \rightarrow X^{*}$ such that the span of $A$ is dense, $x \circ g \in L_{1}(\mu)$ for every $x \in A$, but $g$ is not $w^{*}$-integrable.

REMARK 3.2. Equivalences (a)-(d) are well-known (perhaps except the fact that we may choose the operator Tauberian). (e) is new as far as I know.

The separable quotient problem is extremely difficult as is illustrated by the following result (see $[\mathrm{Mu}]$ ): A real Banach space has a separable infinite-dimensional quotient whenever its dual has an infinite-dimensional subspace which is either reflexive or isomorphic to $c_{0}$ or $\ell_{1}$. 
4. Thickness and a problem from function theory. Recall Marshall's theorem: The norm-closed convex hull of the Blaschke products is the unit ball of $H^{\infty}(D)$ (Here $D$ is the open unit disk in the complex plane). See [Gar] for definitions of the terms we use here as well as for a proof of Marshall's theorem.

It is well-known that $H^{\infty}(D)$ may be realized as the dual of $L^{1}(D) / H_{0}^{1}(D)$. From results of Fernandez, Hui and Shapiro (see [FHS] and [Sh]) we know that the set of Blaschke products are of first Baire category in $H^{\infty}(D)$ and fulfills property (c) of Theorem 1.5. All that is written above is also true with inner functions instead of Blaschke products.

Note that the inner functions is not a James boundary, they form a "small" part of the $w^{*}$-exposed points of $B_{H^{\infty}(D)}$. This can be seen from a paper of R.P. Phelps $([\mathrm{Ph}])$, where it is shown that in $H^{\infty}(D)$ every exposed point of the unit ball is $w^{*}$-exposed and that the set of exposed points is the set of norm one functions $f$ for which $\left\{t \in \partial D:\left|f\left(e^{i t}\right)\right|=1\right\}$ has positive Lebesgue measure. Thus, the result of Fernandez, Hui and Shapiro does not follow from Theorem 2.3 nor Theorem 2.4.

The open problem, posed in [FHS] is whether the inner products or the Blaschke products fulfills property (c) of Theorem 1.1. This would, if it is true, be a considerable strengthening of Marshall's theorem. By Theorem 1.1 we now give equivalent formulations of the open problem:

Problem 4.1. Let $A$ be the set of inner functions or the Blaschke products in $H^{\infty}(D)$. The following questions are open and equivalent:

(a) The set $A$ is thick.

(b) Whenever a sequence of functionals in $H^{\infty}(D)^{*}$ is pointwise bounded on $A$, it is bounded in norm.

(c) Whenever a continuous linear Tauberian injection from a Banach space $X$ is onto $A$, then this operator is onto $H^{\infty}(D)$.

(d) The span of $A$ is barrelled (it is dense by Marshall's theorem).

(e) Whenever $(\Omega, \Sigma, \mu)$ is a measure space and a $w^{*}$-measurable function $g: \Omega \rightarrow$ $H^{\infty}(D)^{*}$ is such that, for all $x \in A, x \circ g \in L_{1}(\mu)$, then $g$ is $w^{*}$-integrable.

Deep results on $H^{\infty}(D)^{*}$ are given in [B].

\section{REFERENCES}

[AnP] T.A. Abrahamsen, M. Põldvere and O. Nygaard, On weak integrability and boundedness in Banach spaces, J. Math. Anal. Appl. 314 (2006), 67-74.

[AN] W. ARENDT AND N. NikOLSKI, Vector-valued holomorphic functions revisited, Math. Zeitschrift 234 (2000), 777-805.

[BK] G. Bennett and N.J. Kalton, Inclusion theorems for $K$-spaces, Canad. J. Math. 25 (1973), 511-524.

[B] J. Bourgain, New Banach space properties of the disc algebra and $H^{\infty}$, Acta. Math. 152 (1984), 1-48. 
[D] J. Diestel, Sequences and Series in Banach Spaces, Graduate Texts in Mathematics Vol. 92, Springer-Verlag, Berlin - Heidelberg - New York, 1984.

[DU] J. Diestel And J.J. Uhl, JR., Vector Measures, Math. Surveys, Vol. 15, Amer. Math. Soc., 1977.

[FHS] J. FERnAndez, S. Hui, AND H. Shapiro, Unimodular functions and uniform boundedness, Publ. Mat. 33 (1989), 139-146.

[FZ] M. FABIAN AND V. ZiZleR, Introduction to Banach spaces III, Charles Univerity, Prague, 1997.

[Fo1] V.P. Fonf, Weakly extremal properties of Banach spaces, Mat. Zametki 45(6) (1989), 83-92; English translation in: Math. Notes 45 (1989), 488-494.

[Fo2] On exposed and smooth points of convex bodies in Banach spaces, Bull. London Math. Soc. 28 (1996), 51-58.

[FL] V.P. Fonf AND J. Lindenstrauss, Boundaries and generation of convex sets, Israel. J. Math. 136 (2003), 157-172.

[Gar] J. Garnett, Bounded analytic functions, Academic Press, New Jersey, 1981.

[KF] M.I. Kadets And V.P. Fonf, Two theorems on massiveness of a boundary in reflexive Banach space, Funct. Anal. Appl. 17 (1983), 77-78.

[KMS] P.S. Kenderov, W.B. Moors And ScotT Sciffer, Norm attaining functionals on $C(T)$, Proc. Amer. Math. Soc. 126(1) (1998), 153-157.

[Mu J. MujicA, Separable quotients of Banach spaces, Rev. Mat. Comp. Madrid 10 (1996), 299-330.

[N1] O. NygaARd, A strong Uniform Boundedness Principle in Banach spaces, Proc. Amer. Math. Soc. 129 (2001), 861-863.

[N2] Boundedness and surjectivity in normed spaces, Int. J. Math. Math. Sci. 32 (2002), 149-165.

[N3] , Some dual Tauberian embeddings, Acta Comment. Univ. Tartu. Math. 5 (2001), 35-42.

[Ph] R.P. PhelPs, Extreme points in function algebras, Duke. Math. J. 32 (1965), 267-277.

[R2] W. Rudin, Functional Analysis, 2nd ed., McGraw-Hill International Book Company, New York - London - Toronto, 1991.

[R1] , Real and Complex Analysis, 3rd ed., McGraw-Hill International Book Company, New York - London - Toronto, 1987.

[Ru] W. RuEss, Duality and geometry of spaces of compact operators, Proc. 3rd Paderborn Conf. Funct. Analysis, North-Holland Math. Studies Vol. 90, (1984) 59-78.

[SW] S. SAXon And A. Wilansky, The equivalence of some Banach space problems, Colloq. Math. 37 (1977), 217-226.

[Sh] H.S. Shapiro, A uniform boundedness principle concerning inner functions, $J$. Analyse Mat. 50 (1988), 183-188.

[S] S. Simons, An Eigenvector Proof of Fatou's Lemma for Continuous Functions, The Math. Int. 17(3) (1995), 67-70. 
[Y] K. YosidA, Functional Analysis, 6th ed., Springer Verlag, Berlin - Heidelberg New York, 1980.

Received 17 February, 2005 and in revised form 25 August, 2005. 